MATEC Web of Conferences 34, 01006 (2015)

DOI: $10.1051 /$ matecconf/ 20153401006

(C) Owned by the authors, published by EDP Sciences, 2015

\title{
PERFORMANCE OF NANOCOMPOSITE CERAMICS BY WIRE ELECTRICAL DISCHARGE MACHINING
}

\author{
Chengmao Zhang ${ }^{1, a}$ \\ ${ }^{1}$ Linyi University, Shandong, China
}

\begin{abstract}
A new process of machining the TiN/Si3N4 nanocomposite ceramics using wire electrical discharge machining is proposed in this paper. The process is able to effectively machine a large surface area on the TiN/Si3N4 nanocomposite ceramics with good surface quality and low cost. The effects of machining conditions on the material removal rate, and surface roughness have been investigated. The surface microstructures machined by the new process are examined with a scanning electron microscope (SEM). The results show that the TiN/Si3N4 nanocomposite ceramics is removed by melting, evaporation and thermal spall. The experimental results indicate that the cutting speed (material removal rate), the surface roughness and the width of the slit of cutting test material significantly depend on experiment parameters. An appropriate servo voltage, a short pulse-on time, and a short pulse-off time, which are normally associated with a high cutting speed, have little effect on the surface roughness. During experiments, parameters such as open circuit voltage, pulse duration and dielectric fluid pressure were changed to explore their effect on the surface roughness.
\end{abstract}

\section{INTRODUCTION}

WEDM was first introduced to the manufacturing industry in the late 1960s.It is considered as a unique adaptation of the conventional EDM process, which uses an electrode to initialise the sparking process. However, WEDM utilises a continuously travelling wire electrode made of thin copper, molybdenum or tungsten of diameter $0.05-0.3 \mathrm{~mm}$ [1], which is capable of achieving very small corner radii. The wire is kept in tension using a mechanical tensioning device reducing the tendency of producing inaccurate parts. During the WEDM process, the material is eroded ahead of the wire and there is no direct contact between the work-piece and the wire, eliminating the mechanical stresses during machining. In addition, the WEDM process is able to machine exotic and high strength and temperature resistive (HSTR) materials and eliminate the geometrical changes occurring in the machining of heat-treated steels [2].

There has been a great upsurge of interest in ceramic materials over the last few years. As a result of this interest in ceramic materials, significant advances in use and development have been made. Ceramics are used more and more in engineering industry 、 space technology and oil patch as a kind of materials for its high intensity 、 low density 、 high hardness 、 low expansion etal [3-9]. But they are very difficult to machine because of their low reliability and hard brittle. Recently, Si3N4 - TiN composites have received much attention because of the possibility for electrical discharge machining [10]. Nanocomposite ceramics have

\footnotetext{
a Corresponding author: 1ydxzcm@126.com
}

excellent mechanical properties at both ambient 、 elevated temperatures and good electrical conductivity, they are called "omnipotence materials "or "materials of 21 century". So the investigation of machine nanocomposite ceramic by wire electrical discharge machining (WEDM) is very important [11].

Since WEDM has been shown to be an economical and effective technique for machining difficult-to-cut materials, the development of the process for electromachining the TiN/Si3N4 nanocomposite ceramics is an important research topic in the field of nontraditional machining. The topic of how to reduce machining time and to maintain reasonable accuracy has always been of research interest. This paper proposes a new technique of machining the TiN/Si3N4 nanocomposite ceramics using WEDM. The effects of machining conditions on the material removal rate (MRR), and surface roughness (Ra) have been investigated. In addition, the machined surfaces by the new process are examined with a scanning electron microscope (SEM), an energy dispersive spectrometer (EDS), and an X-ray diffraction (XRD). Through the comprehensive analysis of the test results, the effect law of the process parameters on the technical index of WEDM is concluded, which provides the basis for parameters selection in WEDM.

\section{PRINCIPLE FOR WEMD OF NANOCOMPOSITE CERAMICS}

The principle for WEDM of the TiN/Si3N4 nanocomposite ceramics is illustrated in Fig.1. WEDM 
machine (DK7732, Suzhou new Spark Machine Tool Co., Ltd.) is showed as Fig.2. The wire electrode and the work-piece are connected to the negative and positive poles of the Impulsing power, respectively. The wire electrode is molybdenum wire with the diameter of $0.18 \mathrm{~mm}$. The wire electrode is mounted onto a rotary spindle, driven by an A.C. motor. The work-piece is bar with dimensions of $3 \mathrm{~mm} \times 4 \mathrm{~mm} \times 40 \mathrm{~mm}$ and is mounted on to a numerically controlled (NC) table. The machining fluid is a water-based emulsion.

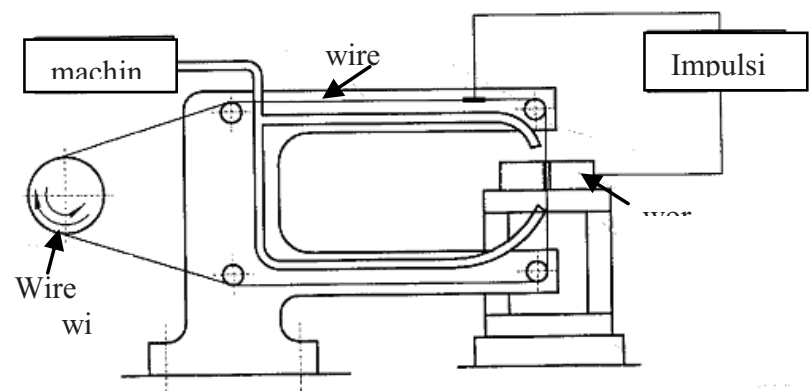

Fig 1. sketch map of WEDM

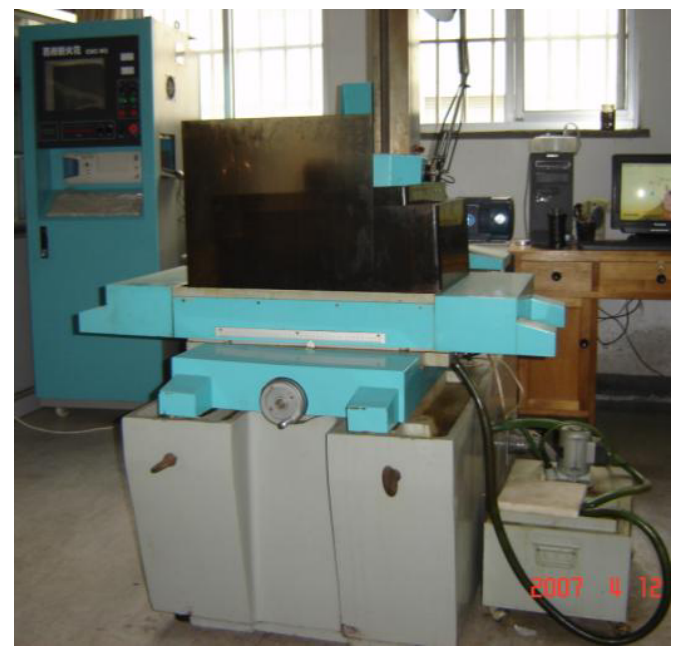

Fig 2. WEDM machine

During machining, the wire electrode rotates at a high speed, the TiN/Si3N4 nanocomposite ceramics workpiece is fed towards the wire electrode driven by a D.C. motor. As short circuits or arcs are generated in WEDM, the work-piece is fed back by the D.C. motor. After short circuits or arcs are cleared up, the work-piece is fed on again. The machining fluid is flushed into the gap between the wire electrode and the work-piece with several nozzles. As the work-piece approaches the wire electrode and the distance between the work-piece and the electrode reaches the discharge gap, electrical discharges occur from the edge and the bottom of the electrodes. The instantaneous high temperature and pressure plasma cause $\mathrm{TiN} / \mathrm{Si} 3 \mathrm{~N} 4$ nanocomposite to be removed by WEDM. The wire electrode are fixed on the wire winding drum, the working fluid is flushed into the gap largely, the chips are flushed away easily, the wire electrodes and the work-piece are cooled quickly; therefore WEDM improves the stability of the electrical discharges. The wire electrode rotates to ensure that the electrodes are worn homogeneously. In addition, using molybdenum wire as the electrode, the wire electrode is manufactured easily and shows low cost.

\section{EXPERIMENTAL PROCEDURES}

In the following experiments, the work-piece material was the TiN/Si3N4 nanocomposite ceramics, the wire electrode is molybdenum wire with the diameter of $0.18 \mathrm{~mm}$. The wire electrode feed speed was $10 \mathrm{~m} / \mathrm{s}$. The machining fluid was composed of 20 mass $\%$ emulsified soap (NG-1, Nanjing Nanguang electric processing new technology research institute, China) and 80 mass \% distilled water, which were mixed with a constant speed power-driver mixer.

The material removal rate (MRR) was obtained through measuring the average processing area within a minute. The surface roughness (SR) was measured by a surface roughness tester. The microstructure of the workpiece surface was examined with a scanning electron microscope (SEM), an energy dispersive spectrometer (EDS), and an X-ray diffraction (XRD). All the observed specimens had been cleaned ultrasonically and dried with a hot-air blower before the examination.

\section{RESULTS AND DISCUSSION}

\subsection{Effect of the pulse-on time on cutting speed and the width of slit}

This work employed WEDM equipment; the increases in the peak current with pulse-on time increased, indicates the performance of the circuit design. Fig. 3 plots the cutting speed versus the pulse-on time for the TiN/Si3N4 nanocomposite ceramics by the WEDM. These experimental results indicate that MRR increases with increasing pulse-on time. Fig. 4 plots the width of the slit against pulse-on time for the TiN/Si3N4 nanocomposite ceramics material. The experimental results reveal that the width of the slit increases with increasing pulse-on time. The narrowest slit was obtained with the TiN/Si3N4 nanocomposite ceramics material. These results may be attributed to the fact that the thermal conductivity and electrical conductivity of material are lower, and that the melting point of Si3N4 particles is high, leading to the requirement for much more thermal energy per unit volume to melt composite material. Therefore, the slit is narrower[12].

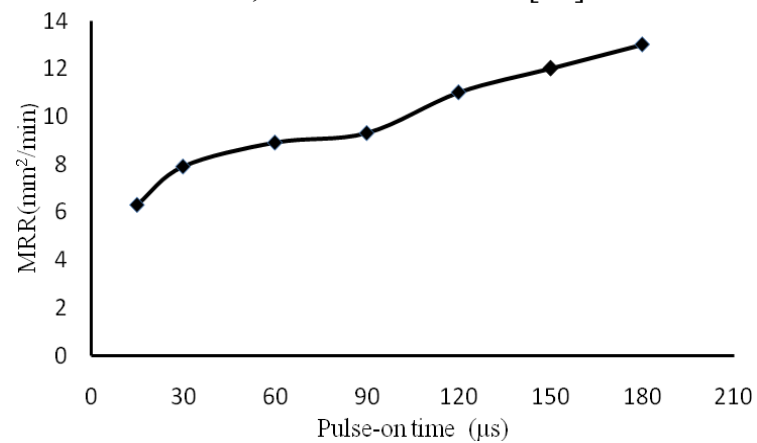

Fig 3. Pulse-on time versus cutting speed 


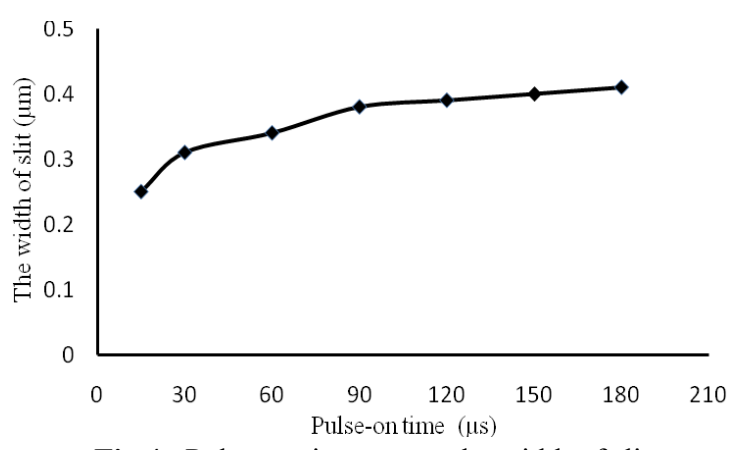

Fig 4. Pulse-on time versus the width of slit

\subsection{SEM observation of the machined surface}

The scanning electron microscope (SEM) micrographs of the unmachined and machined surface with different processing parameters are illustrated in Fig.5 and fig.6. Many spark-induced craters, melt-formation droplets and micropores exist in these machined micrographs. The phenomena indicate that the TiN/Si3N4 nanocomposite ceramics is molten or evaporated by the sparking thermal energy. During machining, sparks are formed at the electrical conductive phase on the TiN/Si3N4 nanocomposite ceramics. The discharged energy produces very high temperatures at the point of the spark, causing a minute part of the work-piece to melt and vaporize. As the spark ceases, a violent bubble causes superheated, molten liquid on the work-piece surface to explode into the gap. Most molten material is flushed away to form craters on the surface. However, not all of the molten material can be removed because of the surface tension, tensile strength, and bonding forces between liquid and solid. The molten material remained on the surface of work-piece is cooled by the working fluid, and some droplets are formed. The formation of micropores is ascribed to the ejection of gases that escape from the solidified material.

It is obvious that the craters are bigger and deeper in case of five power transistors increases. when compared to one power transistor under the same conditions. Stronger wire electrons bombardment results in biggerand deeper craters, leading to a rougher surface. This observation is in accordance with the roughness values.

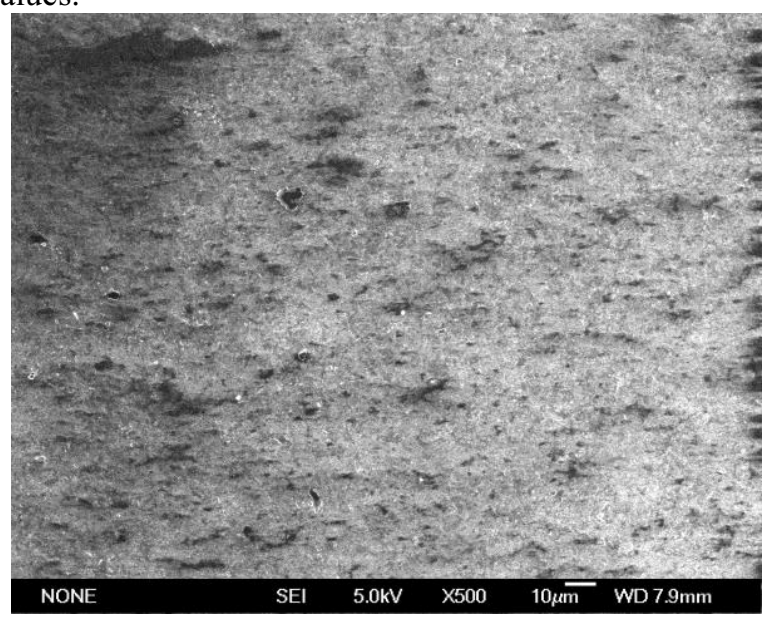

Fig 5. SEM micrographs of un-WEDMed TiN/Si3N4 nanocomposite ceramics surface

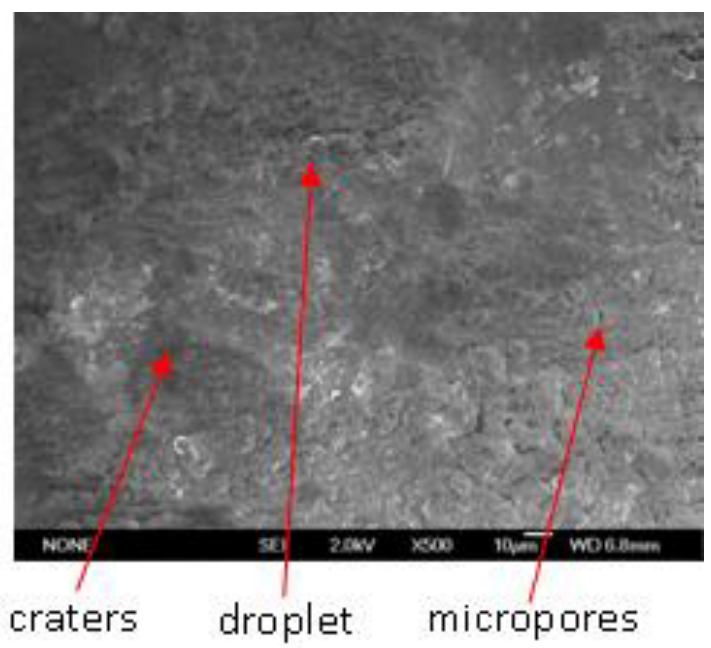

(a) one power transistor

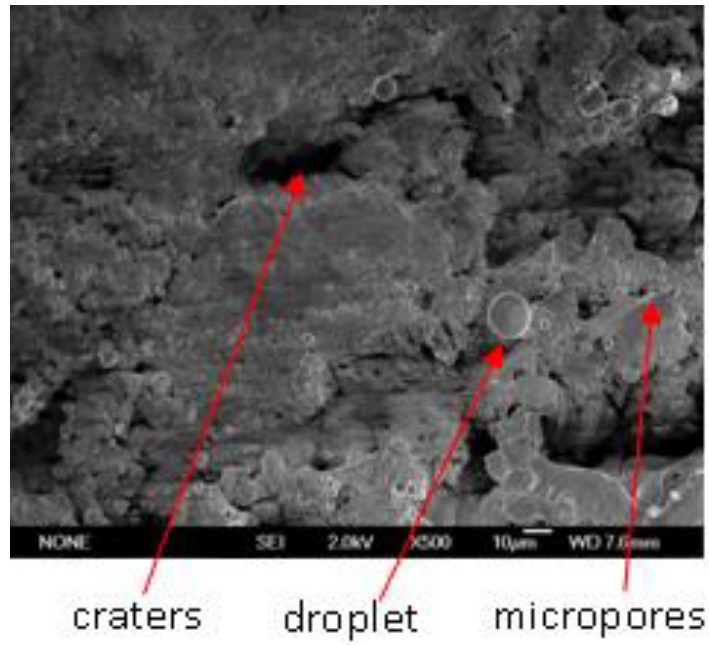

(b) five power transistors

Fig 6. SEM micrographs of WEDMed TiN/Si3N4 nanocomposite ceramics surface

The machined surfaces of the TiN/Si3N4 nanocomposite ceramics sample in different machining conditions are observed under SEM with higher magnification. Fig. 6(a) and (b) shows the typical micrographs obtained from the observation. It is evident that the surface microcracks are observed on the machined surface. The microcracks formation is associated with the development of high thermal stresses during machining, and it is responsible for the thermal spalling removal of the TiN/Si3N4 nanocomposite ceramics.

In the WEDM process, the TiN/Si3N4 nanocomposite ceramics goes through thermal cycling, so a complex temperature gradient is established. On sudden heating, local compressive shear stresses develop because the expanding material is prevented from doing so by the cooler interior material. At the same time, this places the interior material in tension as it is pulled by the outer material as it tries to expand. This situation is reversed on sudden cooling. The TiN/Si3N4 nanocomposite ceramics work-piece is subjected to higher stress due to more severe gradients. When the degree of induced stress exceeds the maximum tensile strength of the TiN/Si3N4 
nanocomposite ceramics, cracking occurs on the machined surface. With extreme hardness and brittleness, the TiN/Si3N4 nanocomposite ceramics tends to promote the formation of steep temperature gradients away from the melting and evaporation zone, so the microcracks are easily produced, which leads to thermal spalling of the TiN/Si3N4 nanocomposite ceramics during machining. The removal mechanism in WEDM of the TiN/Si3N4 nanocomposite ceramics consists of not just the melting and evaporation but also thermal spalling.

It can also be seen from Fig. 6 that the number and length of the microcracks on the machined surface increase with the power transistor numbers increases. The discharge energy delivered to the machining gap increases with the power transistor numbers increases, the heating and cooling effect is enhanced, the temperature gradient and stress during machining increase, so the number and length of the microcracks on the surface increase with the power transistor numbers increases.

\subsection{The influence of machining parameters on surface roughness}

Figs.7 and 8 show surface roughness versus pulse duration and open circuit voltage after WEDM, respectively. According to these figures, the surface roughness increased when the pulse duration and open circuit were increased. The surface roughness is obviously affected by the amount of discharge energy and on-time, since the greater the discharge energy conducted into the machining zone, the greater the melted depth of the work-piece that is created. Furthermore, greater discharge energy will produce a larger crater, causing a larger surface roughness value on the work-piece. As a consequence, different surface roughness can be expected for both microstructures for identical discharge condition[13].

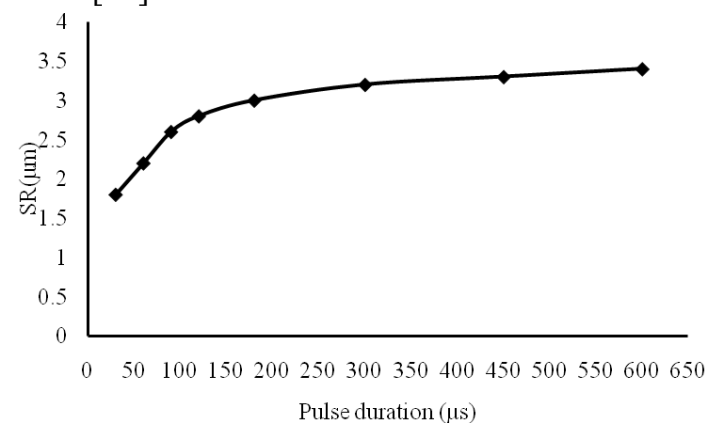

Fig 7. Surface roughness Ra vs. pulse duration

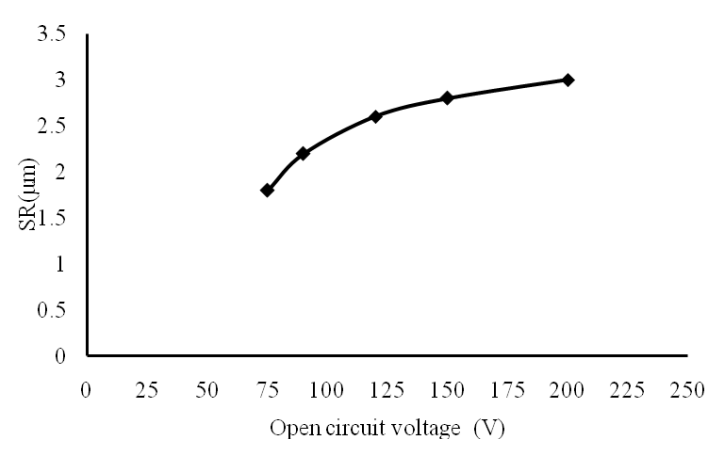

Fig 8. Surface roughness Ra vs. open circuit voltage
Fig.9 shows surface roughness versus the dielectric fluid pressure. As seen from this figure, the surface roughness decreased slightly with increasing the dielectric fluid pressure in chosen conditions. This result can be explained by the cooling effect of increasing dielectric fluid flow on the work-piece surface. Another possible reason may be that increased the dielectric fluid flow prevent the debris to adhere to surface. In addition, the cutting performance with increasing the dielectric fluid pressure has been also improved since the removed particles in the machining gap are evacuated more efficiently.

Fig.10 shows the machined surface roughness versus pulse-on time for the TiN/Si3N4 nanocomposite ceramics. The Ra value remained almost constant as the pulse on time increased. Additionally, for the TiN/Si3N4 nanocomposite ceramics material, a pulse on time of $15 \mu_{\mathrm{S}}$ yielded a relatively fine surface roughness perhaps because the material contained an appropriate quantity of reinforcing Si3N4 particles, with both low thermal conductivity and low electrical conductivity, such that the use of high discharge energy may prevent the enlargement of discharge craters, resulting in the best surface roughness. Compares the SEM micrographs of the surface integrity for the TiN/Si3N4 nanocomposite ceramics material under pulse-on times set to 15 and $180 \mu_{\mathrm{s}}$ after WEDM machining. The figure shows that the surface roughness of the TiN/Si3N4 nanocomposite ceramics material increases with increasing pulse on time. Increasing the pulse on time generates high discharge energy, widening and deepening discharge craters of work-piece surface [14], so deteriorated the surface integrity.

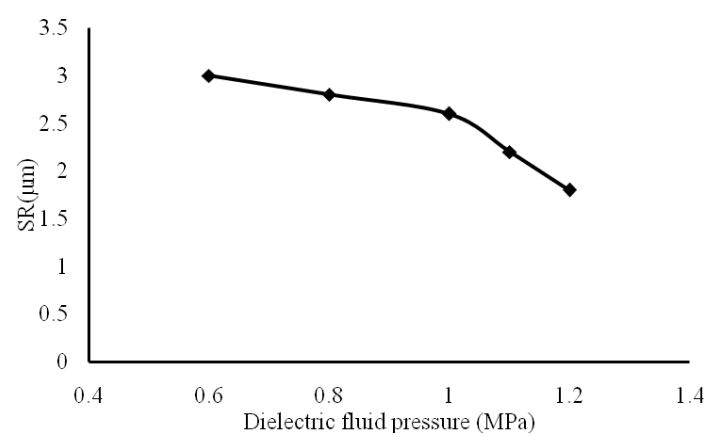

Fig 9. Surface roughness Ra vs. dielectric fluid pressure

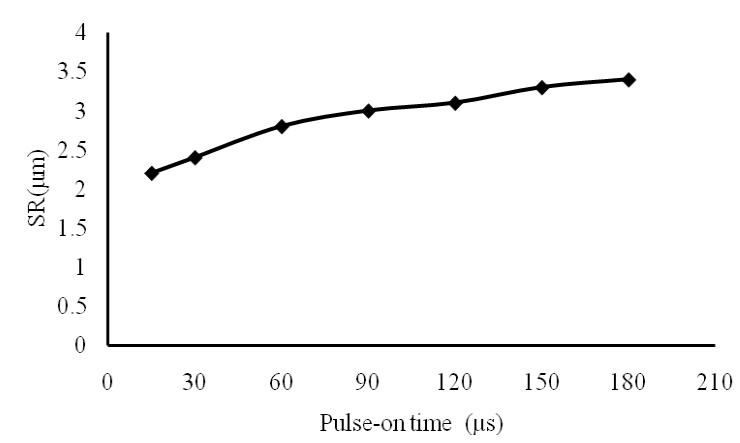

Fig 10. Pulse-on time versus surface roughness 


\section{CONCLUSIONS}

(a) The narrow width of slit led to difficulty in repelling debris from the craters of machined surface into the dielectric.

(b) The machined surfaces are characterized by craters, droplets, micro-pores and micro-cracks. The removal mechanism during WEDM of the TiN/Si3N4 nanocomposite ceramics consists of melting, evaporation and thermal spall.

(c) The surface roughness increased when the pulse duration and open circuit voltage were increased. It appears that the surface roughness primarily depends on these parameters, dielectric fluid pressure and wire speed not seeming to have much of influence.

\section{ACKNOWLEDGMENTS}

Lian Gao,Xihai Jin provided the TiN/Si3N4 nanocomposite ceramics of State Key Lab on High Performance Ceramics and Superfine Microstructure, Shanghai Institute of Ceramics, Chinese Academy of Sciences. And all those who contributed directly or indirectly are thanked. This work is supported by A Project of Shandong Province Higher Educational Science and Technology Program (Grant No. J14 LB59) and A Project of Linyi 2014 Science and Technology Program (Grant No. 201414028).

\section{REFERENCES}

1. D.Rakwal and E. Bamberg, Slicing, Cleaning and Kerf Analysis of Germanium Wafers Machined by Wire Electrical Discharge Machining, J. Mater. Process. Technol. 8, 3740-3751(2009)

2. K.H.Ho, S.T. Newman,S. Rahimifard, R.D. Allen. State of the art in wire electrical discharge machining (WEDM). International Journal of Machine Tools \& Manufacture. 44,1247-1259(2004)

3. Guo XZ, Yang H, Zhang LJ, Zhu XY. Sintering behavior, microstructure and mechanical properties of silicon carbide ceramics containing different nano-TiN additive. Ceram Int 2010;36,161-5(2010)

4. Simba BG, Santos C, Bondioli MJ, Strecker K, Lima ES, Prado da Silva MH. Strength improvement of LPS$\mathrm{SiC}$ ceramics by oxidation treatment. Int $\mathrm{J}$ Refract Met Hard Mater 2010;28,484-8(2010)

5. Margiotta JC, Zhang DJ, Nagle DC. Microstructural evolution during silicon carbide ( $\mathrm{SiC}$ ) formation by liquid silicon infiltration using optical microscopy. Int J Refract Met Hard Mater 2010;28,191-7(2010)

6. Strecker K, Ribeiro S, Oberacker R, Hoffmann MJ. Influence of microstructural variation on fracture toughness of LPS-SiC ceramics. Int J Refract Met Hard Mater 2004;22,169-75(2004)

7. Yang XH, Zhang YM, Han JC. High speed lapping of $\mathrm{SiC}$ ceramic material with fixed abrasive. Key Eng Mater 336-338,1458-60(2007)

8. Shumyacher VM, Dushko OV, Pushkarev DO. Predicting the grinding efficiency of hard ceramics in terms of surface brittleness. Russ Eng Res 2009;29,6234(2009)

9. Agarwal S, Rao PV. Experimental investigation of surface/subsurface damage formation and material removal mechanisms in $\mathrm{SiC}$ grinding. Int J Mach Tools Manuf 2008;48,698-710(2009)

10. Lee, B. T., Yoon, Y. J. and Lee, K. H., Microstructural characterization of electroconductive Si3N4-TiN Composites. Mater. Lett., 47, 71-76(2001)

11. Lian Gao, Jingguo Lia, Takafumi Kusunoseb, Koichi Niiharab. Preparation and properties of $\mathrm{TiN}-$ Si3N4 composites. Journal of the European Ceramic Society. 2004,24,381-386(2004)

12. Biing Hwa Yan, Hsien Chung Tsai, Fuang Yuan Huang, Long Chorng Lee. Examination of wire electrical discharge machining of $\mathrm{Al} 2 \mathrm{O} 3 \mathrm{p} / 6061 \mathrm{Al}$ composites. International Journal of Machine Tools \& Manufacture, 45, 251-259 (2005)

13. Ahmet Hasçalýk, Ulas Çaydas. Experimental study of wire electrical discharge machining of AISI D5 tool steel, Journal of Materials Processing Technology, 148, 362-367(2004)

14. N.P. Hung, L.J. Yang, K.W. Leong, Electrical discharge machining of cast metal matrix composites, Journal of Materials Processing Technology 44, 229-236 (1994) 